\title{
Extra-cardiac manifestations of adult congenital heart disease
}

\author{
Stephen A. Gaeta, MD, $\mathrm{PhD}^{a}$, Cary Ward, $\mathrm{MD}^{a}$, and \\ Richard A. Krasuski, MD ${ }^{a, b, *}$
}

${ }^{a}$ Division of Cardiology, Duke University Medical Center, Durham, NC

${ }^{\mathrm{b}}$ Duke Clinical Research Institute, Durham, NC

\begin{abstract}
A B S T R A C T
Advancement in correction or palliation of congenital cardiac lesions has greatly improved the lifespan of congenital heart disease patients, resulting in a rapidly growing adult congenital heart disease (ACHD) population. As this group has increased in number and age, emerging science has highlighted the systemic nature of ACHD. Providers caring for these patients are tasked with long-term management of multiple neurologic, pulmonary, hepatic, renal, and endocrine manifestations that arise as syndromic associations with congenital heart defects or as sequelae of primary structural or hemodynamic abnormalities. In this review, we outline the current understanding and recent research into these extra-cardiac manifestations.
\end{abstract}

Key words: Cardiovascular disease, Adult congenital heart disease, Pulmonary hypertension.

(c) 2016 Elsevier Inc. All rights reserved.

Advances in the diagnosis and management of congenital heart disease (CHD) over the past several decades have greatly improved longevity and quality of life in the CHD population. Palliative or corrective interventions are now available for nearly every type of congenital cardiac lesion, and approximately $90 \%$ of affected children now survive to adulthood [1]. Adults with CHD (ACHD) already outnumber pediatric CHD patients, and this population is expected to grow by $5 \%$ per year [1,2]. Strikingly, the median age of severe CHD patients has increased from 11 years in 1985 to 25 years in 2010 [3]. Continued improvement in CHD interventions means that new (and more complex) populations will likely reach adulthood in the coming years.

Practitioners who take care of adults with CHD are often charged with the delivery or coordination of comprehensive care for these complex patients, including the management of manifestations and complications that arise later in life. ACHD has many extra-cardiac effects, both as syndromic associations with congenital heart defects, or more commonly, as sequelae of primary structural or hemodynamic abnormalities. The role of genetic predilection remains undefined for nearly all extra-cardiac complications, though their incidence has clearly risen over the past decades. Indeed, more than a third of hospital admissions for ACHD patients are now for noncardiovascular reasons [4]. Optimal care of ACHD patients, therefore, mandates a thorough understanding of its multiorgan system manifestations. Herein, we will review current understanding of the most common manifestations of ACHD organized by the affected organ systems.

\section{Neurologic/neuropsychiatric}

\section{Cerebrovascular complications}

A recent study quantified the strikingly high rates of stroke in ACHD [5]. Among nearly 30,000 ACHD patients, $8.9 \%$ of men

Dr. Krasuski serves a consultant for Actelion and Bayer and is on the scientific advisory board for Ventripoint. The other authors note no conflicts of interest.

*Correspondence to: Department of Cardiovascular Medicine, Duke University Medical Center, 2301 Erwin Rd, Durham, NC 27710. Tel.: +1 919684 2407; fax: +1 9196817917.

E-mail address: richard.krasuski@duke.edu (R.A. Krasuski). 
and $6.8 \%$ of women suffered a stroke before age 65 , with an incidence of ischemic and hemorrhagic stroke 12-fold and 6-fold higher than the general population. Ischemic stroke was associated with heart failure, diabetes, and recent MI; CHD lesions predisposing to cyanosis and left-sided CHD lesions had the highest associated stroke rate.

Mechanistically, CHD can directly increase stroke risk through right-to-left shunting, allowing paradoxical embolism of thrombus from venous to arterial circulations. This is most commonly seen in the context of an atrial septal defect (ASD) or patent foramen ovale (PFO), particularly when associated with atrial septal aneurysm, but can also occur with shunting through baffle leaks in d-transposition of the great arteries (TGA) repaired by atrial switch (Mustard or Senning procedures) or in palliated single-ventricle patients with surgical fenestrations. In the presence of these defects, right-to-left shunting can occur due to pathologically elevated right atrial (RA) pressure or with transient physiologic elevation of RA pressure to greater than left atrial (LA) pressure (as with the Valsalva maneuver or briefly during early ventricular systole) [6]. Patients with Ebstein anomaly commonly have associated ASD and are at theoretically higher risk of paradoxical embolism due to blood stasis within an enlarged RA [7]. Current guidelines recommend closure of ASD (either percutaneously or surgically) and repair of baffle leaks following paradoxical embolism [7]. The benefit of PFO closure in cryptogenic stroke remains strongly debated and will not be discussed. Interested readers are referred to Rohrhoff et al. [8] for additional information.

Cyanotic congenital heart disease (CCHD) patients are at high risk for iatrogenic stroke due to paradoxical embolization of air or thrombus. As such, indwelling venous catheters should be avoided and if necessary precautions taken to avoid introducing air (including use of filters). Specific pathophysiology of stroke in CCHD is discussed below.

The reason for higher rates of stroke with left-sided lesions (including congenital mitral stenosis, mitral insufficiency, aortic stenosis, aortic insufficiency, and aortic coarctation) is unclear, but may be due to a predisposition to arterial thromboembolism. Regardless of the specific lesion, CHD can predispose to arterial thrombi through heart failure, atrial arrhythmias, mechanical valve prostheses, cardiothoracic surgery, and catheter-based interventions.

\section{Intracranial aneurysms}

Intracranial aneurysms (IA) are five times more common in coarctation of the aorta than the general population, occurring in $\sim 10 \%$ of screened coarcts [9]. This was previously ascribed to syndromic association, though a recent study has shed new light on this relationship [10]. Screening performed in 80 children prior to early coarctation repair (mean age $=2.6$ years) detected no IA. Importantly, no IA was seen at interval screening following repair (mean age $=15.7$ years). This suggests that IA in adults with unrepaired coarctation is at least in part a secondary effect of coarctation (and likely the resulting hypertension), rather than a neurodevelopmental abnormality. Another explanation is a two-hit hypothesis, with developmental abnormalities predisposing to IA formation after exposure to longstanding hypertension.
An association between bicuspid aortic valve (BAV) and IA has also been reported [11], but this has also been questioned by recent data. In a retrospective study of adults treated for IA, the prevalence of BAV (1-2\%) was similar to the general population [12]. There was, however, an increased prevalence (4.7\%) of thoracic aortic aneurysm (TAA). Indeed, the majority of BAV patients with IA in prior studies also had TAA [11]. As with coarctation, the relationship between TAA and IA is likely a manifestation of both developmental predisposition and shared risk factors (hypertension). Although there are no current screening guidelines specific to $\mathrm{CHD}$, these data suggest that cerebral imaging be considered in CHD patients with TAA.

\section{Cerebral complications of cyanosis}

CCHD carries an exceptionally high risk for stroke. Also, 47\% of adult CCHD patients screened with brain MRI had evidence of prior stroke, with $53 \%$ having had multiple events [13]. Importantly, only $13 \%$ of patients reported a history of prior stroke, suggesting a high prevalence of clinically silent events. Among CCHD patients, hypoxemia and complexity of CHD were identified as stroke risks. Contrary to common teaching, polycythemia and hemostatic abnormalities were not associated with stroke risk, consistent with emerging science suggesting hypocoagulability in these patients $[13,14]$.

CCHD also carries an increased risk for brain abscess. Though data are limited, one study estimated a $2 \%$ incidence over 13-years of follow-up. The most commonly associated CCHD lesions were tetralogy of Fallot (TOF $=61.2 \%$ ) and TGA $(9.6 \%)[15,16]$. Right-to-left shunting carries any increased risk of infective endocarditis and may predispose to brain abscess by both allowing arterial bacteremia (due to bypass of the normal phagocytic filtering ability of the lungs) and a focal ischemic nidus for infection (due to hyperviscosity) [16-18].

\section{Neurodevelopmental issues}

Neurodevelopmental abnormalities are common in ACHD, both as innate changes in brain development (as with Down or DiGeorge syndromes) and as acquired abnormalities due to brain injury. Data on cognitive function in ACHD are limited and mixed [19], but a predilection toward neurologic abnormalities in ACHD can be expected given the high frequency of neurodevelopmental abnormalities in pediatric CHD [20]. The prevalence of neurologic abnormalities among school-age CHD patients ranges from $20 \%$ in total anomalous pulmonary venous connection and isolated atrioventricular septal defects, to as high as 70\% in TGA and hypoplastic left heart syndrome [21]. In general, the prevalence and severity of neurodevelopmental impairment increase with lesion complexity [22].

Acquired brain injury has been associated with cardiopulmonary bypass and circulatory arrest during CHD surgery [20]. Developmental abnormalities can also result from disturbed fetal and/or postnatal cerebral blood flow (seen in hypoplastic left heart syndrome and TGA), hypoxemia (CCHD), failure to thrive, arrhythmias, seizures, or hypotension (often in relation to procedures) [23]. 


\section{Neuropsychiatric}

Psychiatric illness is common in ACHD, perhaps secondary to the myriad psychosocial challenges related to CHD and its treatment $[24,25]$. Up to one-third of North American ACHD patients have low mood or anxiety disorders, many of which are unrecognized by health care providers [25]. Data on this subject are mixed, with the association less clear among European patients $[25,26]$. Importantly, major adverse cardiac events were 2-fold higher over 5 years among depressed ACHD patients [27], and quality of life was more strongly associated with minor depressive symptoms than with exercise capacity [28]. Finally, children undergoing cardiac surgery have high rates of post-traumatic stress disorder, though it remains unknown whether this carries over to adult survivors [29]. ACHD patients should be screened for depression and anxiety, and guidelines recommend assessment of psychosocial needs as part of routine care, with mental health providers included in the care team [20].

Management of ACHD is improved by understanding their psychosocial profile. Adults with complex CHD can exhibit denial or anger regarding their condition. Their concerns frequently include pregnancy and childbirth (including concern over having a child with CHD), limited lifespan, and long-term disability [30]. It is important for providers to recognize and proactively manage these considerations, including routine screening and referral to experienced mental health providers.

\section{Pulmonary}

\section{Pulmonary function limitations}

Abnormal lung function is common in ACHD and is associated with decreased exercise capacity, exercise duration, and worsened functional status. Nearly half (44-47\%) of ACHD patients have restrictive lung disease (RLD), including $89 \%$ following Fontan repair and 76\% with TOF [31,32]. Predictors of RLD include multiple prior thoracotomies (possibly due to associated chest wall deformity or weakness), diaphragmatic nerve palsy, cardiomegaly, atrial arrhythmias (perhaps related to amiodarone use), scoliosis, complex CHD, and increased BMI [32,33]. CCHD and increased CHD complexity are further associated with worsened lung function [33]. RLD can also be seen in CHD associated with lung underdevelopment (as in scimitar syndrome-partial anomalous pulmonary venous return with hypoplastic right lung) or related to impaired pulmonary blood flow due to congenital pulmonic stenosis or TOF [34]. Importantly, moderately to severely impaired lung function (as measured by FVC) is an independent predictor of mortality in ACHD $(\mathrm{OR}=1.6)$ [33].

\section{CHD-associated structural abnormalities}

Enlarged or malpositioned cardiovascular anatomy can directly impinge on adjacent pulmonary structures, leading to altered development or compression. This often manifests early in life when related to congenital malformations, but can theoretically complicate any CHD lesion that results in chamber dilatation. Cardiomegaly (especially right heart enlargement) is a predictor of restrictive lung physiology [33] and is seen in CHD with chamber enlargement due to volume overload (from left-to-right shunts or valvular regurgitation), pressure overload (due to outflow obstruction), or cardiomyopathy. The position of the left atrium beneath the carina allows LA enlargement, in particular, to cause secondary displacement and/or impingement of the mainstem bronchus [35].

Similarly, enlargement or malposition of the great arteries can cause impingement of the mainstem bronchus, leading to tracheobronchomalacia [34]. This is seen early in life in TOF with absent PV, but can theoretically be seen with progressive structural changes in any ACHD patient. Similarly, a pulmonary artery (PA) sling, in which an anomalous left PA arises from the right PA and traverses leftward behind the trachea, can lead to compression of the right mainstem bronchus and distal trachea [35]. In general, such structural cardiopulmonary interactions are well characterized in pediatric patients, but are less appreciated in adults.

\section{Pulmonary hypertension}

Pulmonary hypertension (PH) complicates multiple forms of CHD and carries important prognostic and management implications. The prevalence of $\mathrm{PH}$ varies in different $\mathrm{CHD}$ populations, with estimates from $6 \%$ to $28 \%[36,37]$. $\mathrm{PH}$ confers a higher rate of death $(\mathrm{OR}=2.7)$ and morbidity (including heart failure and arrhythmia; $\mathrm{OR}=3.0$ ) compared to CHD without PH [36]. Moreover, PH patients have higher resource utilization, including higher rates of hospitalization (including intensive care unit stays), echocardiography, and cardiac catheterization [36].

The clinical presentation of $\mathrm{PH}$ is nonspecific, and includes exertional dyspnea, fatigue, chest pain, and syncope [38]. ACHD patients with progressive PH often subconsciously decrease activity and may be unaware of their limitations. There are myriad multiorgan system consequences of $\mathrm{PH}$ including cardiovascular, hematologic, infectious, renal, and hepatic complications [39].

Generally, PH in CHD can be divided into pulmonary venous hypertension, pulmonary arterial hypertension (PAH), or a combination of both. A given anatomical lesion can lead to PH through many different mechanisms, and hemodynamic assessment with direct or indirect measurement of left heart filling pressure is critical to appropriate differentiation [40]. Pulmonary venous hypertension represents passive increase in pulmonary pressure from elevated left-sided filling pressure. This is seen in congenital lesions causing obstruction to left heart inflow (Shone syndrome and cor triatriatum), obstruction to outflow (congenital AS and sub/supravalvular AS, aortic coarctation, Shone syndrome), or CHD-related cardiomyopathy $[37,41]$. PAH, in contrast, arises from structural lesions (including left-to-right shunts) that lead to increased pulmonary vascular resistance. Importantly, PAH can progress even after successful shunt closure.

Not all CHD with systemic-to-pulmonary shunt leads to significant PH. Truncus arteriosus, ventricular septal defect, ASD, and patent ductus arteriosus are the most commonly identified lesions associated with severe PH and Eisenmenger 
syndrome [38]. As compared with atrial-level shunting, ventricular-level shunts cause volume and pressure overload and lead to earlier and more severe $\mathrm{PH}[36,37]$. Shunt-related PAH differs from other etiologies in that chronic RV overload begins early in life, allowing greater compensation (through $\mathrm{RV}$ remodeling) to increased afterload [38].

\section{Hepatic}

There are rare primary hepatic abnormalities that carry an association with CHD $[42,43]$, but the majority of hepatic manifestations in CHD occurs secondary to hemodynamic perturbations and is not usually specific to particular CHD malformations. Cardiac disease of any cause can cause hepatic dysfunction through elevated hepatic vein pressure (congestive hepatopathy), decreased hepatic blood flow (ischemic hepatitis), or hypoxemia [44].

\section{Congestive hepatopathy}

Any elevation in central venous pressure (CVP) is communicated to the liver and can cause hepatic sinusoidal dilatation, edema, and hypertension. Over time, this leads to hepatocyte atrophy, hepatic fibrosis, and portal hypertension [44]. As listed in the Table, increased CVP is encountered in multiple CHD contexts, either through direct elevation of RA pressure [as from left-to-right shunting or tricuspid regurgitation (TR)], secondary elevation of RA pressure due to sub-pulmonic ventricular failure, or following Fontan palliation.

Workup of hepatic dysfunction in CHD includes noninvasive and/or invasive hemodynamic assessment for anatomical causes of increased CVP, including stenosis within surgically created baffles or conduits (as in Fontan, TGA post-Mustard/Senning, or repaired TOF) and worsening valvular stenosis or regurgitation. There is ongoing research into noninvasive measurement of CVP by transient elastography (TE), either by ultrasound or MRI, which has been shown to correlate with CVP and may be useful in management $[45,46]$.

\section{Ischemic hepatitis}

Hypoperfusion from any cause can result in ischemic hepatitis, manifested as hepatocyte necrosis. The majority of ischemic hepatitis occurs in the context of congestive heart failure, in which a combination of poor cardiac output and decreased portal inflow due to hepatic congestion leads to underperfusion [44]. As with congestive hepatopathy, it is not

Table - Congenital heart lesions associated with congestive hepatopathy.

Tricuspid regurgitation (e.g., Ebstein anomaly)

Atrial septal/AV canal defect

Any cause of pulmonary hypertension

Pulmonic regurgitation (e.g., in repaired Tetralogy of Fallot, PH)

Pulmonic stenosis

Systemic ventricular failure

Post-Fontan palliation

Venous baffle stenosis (e.g., repaired D-TGA) specific to CHD type. Rather, it can complicate any CHD presentation that includes poor cardiac output, including cardiomyopathy, hypotension, and arrhythmia (or combination thereof). The biochemical hallmark of ischemic hepatitis is elevation of aminotransferases to the thousands within 24 hours, along with elevations in bilirubin that lag in their rise and fall [42]. Management of ischemic hepatitis is aimed at increasing hepatic perfusion.

\section{Cholelithiasis in CCHD}

Erythrocytosis in CCHD increases bilirubin formation (through heme metabolism) that in turn predisposes to pigment gallstones. Gallstones are twice as prevalent among CCHD (current or previously corrected) compared to agematched acyanotic CHD [47]. Management, as in the general population, should be symptom-driven.

\section{Iatrogenesis}

Hepatic dysfunction in CHD can result from iatrogenesis, especially in the context of medications (drug-related hepatitis) [42]. Of particular concern in ACHD are antiarrhythmic medications, especially amiodarone. Encouragingly, amiodarone-induced hepatotoxicity does not appear to be increased in non-CHD-related right heart failure [48]. Iatrogenic hepatic injury in ACHD can also occur as a result of transfusion-related infections [42]. CHD patients who underwent heart surgery before 1992, when routine hepatitis $C$ virus (HCV) blood screening was instituted, have higher rates of $\mathrm{HCV}$ infection [49]. AHA/ACC guidelines suggest screening such patients for indolent infection.

\section{Cirrhosis}

Chronic congestive hepatopathy from any cause can lead to cirrhosis. Management of cirrhosis in the ACHD population is similar to other patient populations, with focus on reducing hepatic congestion by lowering CVP. Additionally, the hemodynamic effects of cirrhosis itself-decreased systemic vascular resistance, cirrhotic cardiomyopathy, and hypervolemia - can complicate hemodynamic management of ACHD [50]. Theoretically, developing portopulmonary hypertension could further decompensate a cirrhotic ACHD patient. Unfortunately, this entity is both difficult to define and study given myriad etiologies for PH in CHD. Likewise, hepatopulmonary syndrome has never been defined in the CHD population, but its resulting hypoxemia could complicate management of the cirrhotic ACHD patient.

Hepatocellular carcinoma (HCC) can result from any form of advanced liver fibrosis. It has been reported in chronic congestive hepatopathy resulting from Fontan palliation, repaired TOF, and TGA following Mustard repair [50-52]. HCC has not been systematically studied in ACHD patients, and current ACHD guidelines do not address screening.

Combined heart-liver transplant has been performed in end-stage liver disease in CHD, but it is an uncommon procedure performed at limited centers [53-55]. From 1987 to 2010, in the United States, only 97 heart-liver transplants were reported, 17 of which had primary CHD. Further, 5-year 
survival was $72.3 \%$, comparable to that for isolated liver or heart transplantation [56].

Within CHD, the most studied liver disease population is Fontan repairs. These patients are predisposed to hepatic injury by pre-Fontan hypoxemia as well as chronically increased CVP and (often) depressed cardiac output following Fontan palliation [52,57]. The presentation of hepatic dysfunction following Fontan is similar to other congestive hepatopathies, but signs and symptoms may be muted due to chronicity [52]. Additionally, for unclear reasons, the hepatic venous pressure gradient is usually normal [57]. Still, the effects of increased portal pressures, including formation of esophageal varices, can be seen [52].

\section{Renal}

Renal dysfunction is very common in ACHD, with reduced GFR 18-fold more common that the general population. In a cohort of 1102 patients with a broad spectrum of CHD, $50.2 \%$ had at least mild renal dysfunction and 9.3\% had moderately or severely reduced renal function [58]. Renal dysfunction was associated with both simple and complex lesions (Fig. 1). The majority of renal manifestations of ACHD occur secondary to primary cardiac pathophysiology. Syndromic association exists between familial subvalvular and supravalvular aortic stenosis (Williams-Beuren Syndrome) and renal artery stenosis [7,59].

As in any patient, cardiac dysfunction in CHD can lead to secondary renal injury through reduced renal blood flow (low cardiac output), renal venous congestion (elevated CVP), and impaired renal autoregulation. Additionally, nearly all CHD patients are at further risk for renal dysfunction due to adverse exposures including cardiothoracic surgery, cardiopulmonary bypass, and nephrotoxic medications (including ACE-inhibitors and loop diuretics) [58,60].

Renal dysfunction in CCHD is well recognized but poorly understood. Possible pathophysiologic contributions include polycythemia (and subsequent hyperviscosity), chronic hypoxia, changes in intraglomerular hemodynamics, neurohormonal activation, and altered autonomic regulation $[58,60]$.

\section{Renal risk factors}

Predictors of renal dysfunction in ACHD include worsened functional class, diuretic use, and cyanosis. CCHD experiences a nearly 35-fold higher prevalence of renal dysfunction compared to the general population. Patients with Eisenmenger physiology have the lowest glomerular filtration rate and highest prevalence of renal dysfunction (18\%) among ACHD [58]. Notably, prior work has established that nephropathy is higher in unrepaired CCHD, compared to repaired, acyanotic patients, suggesting that cyanosis itself, rather than CHD or its treatment, is largely responsible [61].

\section{Prognostic implications}

Impaired renal function in ACHD is strongly associated with mortality. Also, 6-year mortality with moderately or severely reduced GFR was 5-fold higher than with normal renal

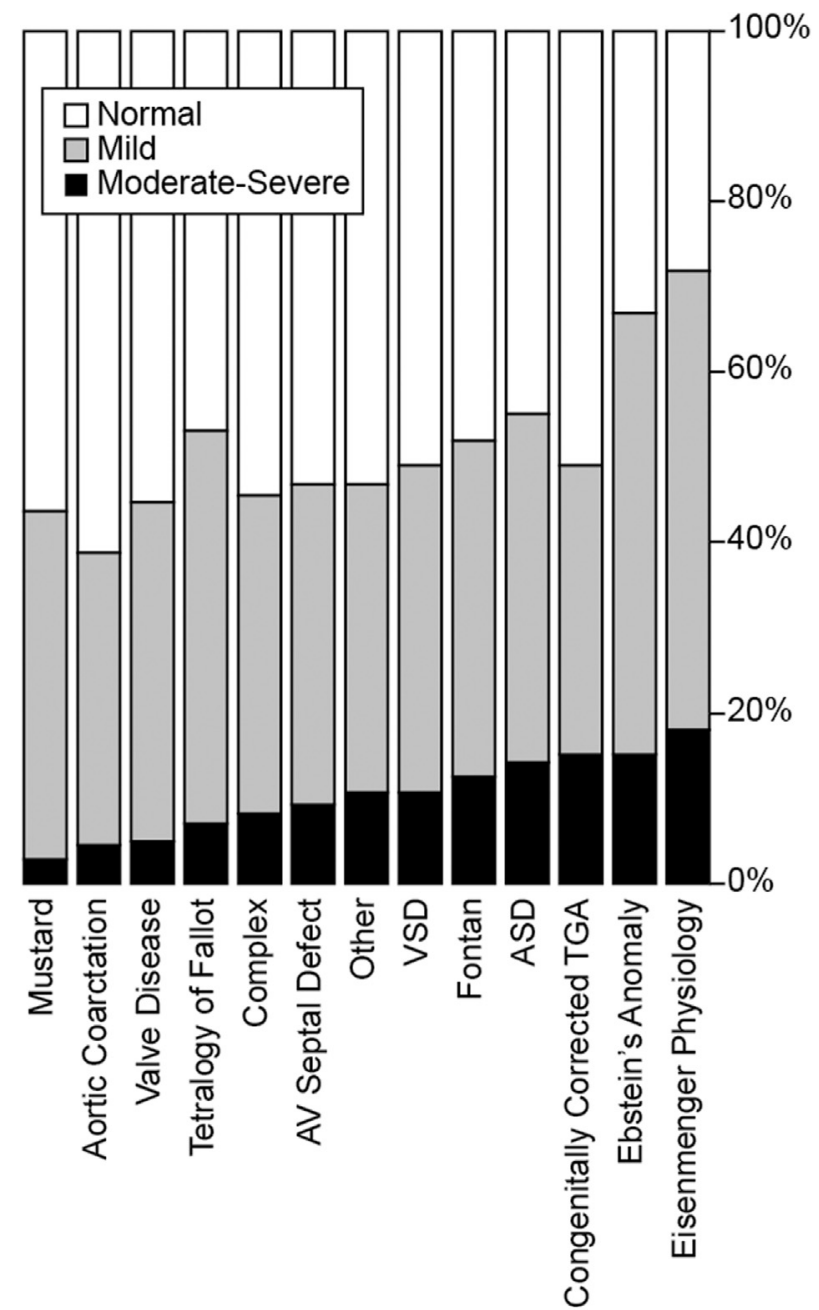

Fig. 1 - Prevalence of normal (white), mildly reduced (gray), or moderately to severely reduced renal function (black) by lesion type. (Adapted with permission from Dimopoulos $\mathrm{K}$ et al. [58])

function (Fig. 2). Notably, renal function is associated with mortality independent of markers of worsening cardiac disease (including functional class and systemic ventricular function), implying it is more than just a marker of advanced disease [58]. Renal dysfunction may drive poorer outcomes by causing volume overload, hypertension, or anemia. Additionally, renal dysfunction increases perioperative risk and impacts both medical and surgical treatment options in CHD [58,61]. Renal and cardiac functions are inextricably linked in advanced CHD, and early detection and management of either may be protective to both (as well as other) organ systems [62].

\section{Management}

Given the high prevalence of nephropathy in ACHD patients, every effort should be made to avoid renal insults. In addition to serial monitoring of renal function (especially in high-risk populations such as CCHD), medications should be reviewed for potential nephrotoxicity. Additionally, adequate prehydration and judicious contrast use should be ensured [7]. 


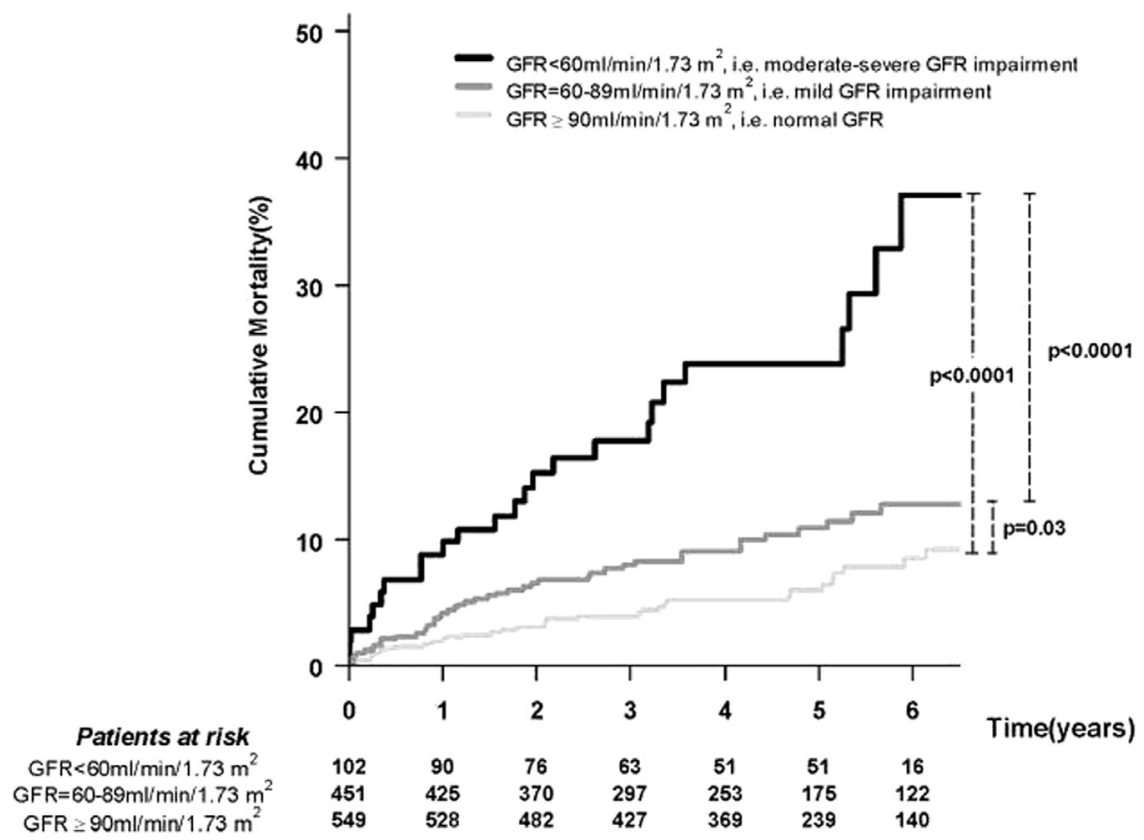

Fig. 2 - Renal dysfunction in ACHD patients is associated with increased mortality risk. (Adapted with permission from Dimopoulos K et al. [58])

Finally, assessment of renal artery anatomy and/or flow should be performed in patients with supravalvular aortic stenosis [7].

There is no management strategy specific to CHD for established renal dysfunction. There are case reports of combined heart-kidney transplant for CHD patients with advanced renal failure, but no systematic study of this strategy has been undertaken $[55,63]$.

\section{Hematologic}

\section{Thrombosis in CHD}

Multiple CHD populations are at higher risk for thrombosis due to hematologic abnormalities, stasis (as in atrial arrhythmias and chamber enlargement), thrombosis of prior interventional sites (stents, grafts, and baffles), and thrombosis due to frequent invasive procedures (catheterization access sites). Moreover, many CHD patients are at higher risk for morbidity or mortality due to thrombosis, for example, due to paradoxical embolization of thrombus (in right-to-left shunts), occlusion of collaterals, or hemodynamic disturbance (e.g., Fontan-palliated patients and those with $\mathrm{PH}$ ). Detailed CHD-specific recommendations regarding prevention and management of thrombosis have been published, but the majority of existing data relates to pediatric populations [64].

\section{Anemia}

Anemia is common in ACHD, with $13 \%$ of non-cyanotic and $29 \%$ of complex non-cyanotic ACHD (TGA and Fontanpalliated patients) affected, respectively $[65,66]$. Anemia in non-cyanotic ACHD is likely multifactorial, with potential mechanisms including iron deficiency, co-existing renal dysfunction, bleeding (either iatrogenic or due to intrinsic coagulopathy), and anemia of chronic disease.

Importantly, anemic non-cyanotic ACHD patients have a 3-fold higher 5-year mortality than non-anemic controls [66]. Although anemia predicts mortality in part as a marker of disease severity (including renal dysfunction), it is also independently associated with mortality [65,66]. For this reason, the cause of anemia in ACHD patients should be determined and managed aggressively.

\section{Hematologic consequences of CCHD}

There are multiple hematologic consequences of CCHDrelated chronic cyanosis, including thrombosis, bleeding, iron deficiency, and hyperviscosity. Defining anemia in CCHD is difficult, as optimal hemoglobin concentration is dependent on degree of hypoxemia [67]. Erythrocytosis and supranormal hemoglobin concentrations result from hypoxemia. Relative anemia, therefore, occurs at much higher hemoglobin levels than in the general population, and often goes unnoticed. Using a definition for anemia corrected for baseline hypoxemia, $61 \%$ of CCHD patients are affected [21].

A major cause of relative anemia in CCHD is iron deficiency. More than a third of CCHD patients are iron deficient [68-70]. Typical indices of iron deficiency including hypochromia and microcytosis are often absent; exclusion of iron deficiency, therefore, requires a complete iron metabolism workup, including serum ferritin and transferrin levels [68]. Importantly, iron deficiency has been identified as a predictor of stroke in CCHD patients, perhaps related to reduced deformability of microcytic red blood cells [71]. For this reason phlebotomy, which can further provoke iron deficiency, is reserved for patients with high hemoglobin and symptoms of 
hyperviscosity (discussed below). Iron deficiency should be avoided in CCHD, with oral iron supplementation as needed to normalize ferritin levels remaining mindful of an overly robust rise in hematocrit [7]. Although physicians are often uncomfortable administering iron in patients with high hematocrit, supplementation has been shown to improve exercise capacity and quality of life in iron-deficient cyanotic patients without increasing hyperviscosity risk $[69,72]$.

CCHD patients have increased pulmonary and cerebrovascular thromboembolic risk [13]. Although impaired clot lysis has been identified as a potential prothombotic mechanism [73], thromboembolic events have not been correlated with hemostatic abnormalities or erythrocytosis [13]. Thrombosis in this population may, therefore, be primarily driven by other elements of Virchow's triad, including stasis due to slow flow in dilated chambers or atrial arrhythmias.

CCHD patients also have a bleeding diathesis, and recent research has identified hypocoagulable hemostatic profiles $[14,73]$. Thromboelastography in cyanotic patients reveals delayed activation of hemostatic mechanisms and slowed clot formation [73], with the degree of coagulation impairment correlated with hematocrit elevations [14]. Indeed, hemostatic improvement was seen with phlebotomy and more impaired coagulation with iron supplementation [14]. Counterintuitively, this suggests a role for phlebotomy in polycythemic CCHD patients preoperatively or during active bleeding $[7,14,64]$.

Acquired von Willebrand syndrome (AVWS) also contributes to the bleeding diathesis of CCHD. A recent study of 221 consecutive ACHD patients found AVWS in $21 \%$ of patients, including $71 \%$ of screened Eisenmenger patients and $29 \%$ of patients with intracardiac shunts [74]. Pre-procedural screening for AVWS should be considered in these subgroups.

CCHD is also associated with thrombocytopenia, and $25 \%$ have platelet counts less than $100 \times 10^{9} / \mathrm{L}$, with counts inversely correlating with hematocrit [75]. Thrombocytopenia may result from decreased fragmentation of megakaryocytes into platelets in the setting of right-to-left shunting. Intact megakaryocytes are able to enter systemic circulation without passing through the pulmonary bed, where they are normally fragmented into platelets. Consistent with this hypothesis, hypoxemia without shunting (e.g., in high altitude environments) does not lead to thrombocytopenia [75].

\section{Hyperviscosity in CCHD}

Extreme erythrocytosis due to chronic hypoxemia in CCHD predisposes to hyperviscosity syndrome, characterized by such nonspecific symptoms as fatigue, headache, visual changes, and dizziness/faintness. Chronically polycythemic patients-especially those with hematocrits $>65 \%$-are at risk for developing symptoms. Acute exacerbations can be provoked by increase in hematocrit, as in dehydration in the setting of systemic illness [76]. Importantly, many of the symptoms of hyperviscosity can also be caused by iron deficiency, mandating a comprehensive workup.

Long-term management of chronically polycythemic patients is unclear. Chronic prevention or treatment of hyperviscosity by phlebotomy must be balanced against the risk of provoking iron deficiency, and current ACHD guidelines do not support routine phlebotomy to prevent hyperviscosity. Rather, therapeutic phlebotomy is reserved only for symptomatic hyperviscosity with hemoglobin $>20 \mathrm{mg} / \mathrm{dL}$ and hematocrit $>65 \%[7,64]$. There are case reports of hydroxyurea treatment, but further study is needed [77].

\section{Endocrine}

There are multiple specific syndromic associations between CHD and endocrinopathy, including Turner syndrome [with estrogen deficiency, diabetes mellitus (DM), and hypothyroidism] [78], DiGeorge (22q11.2 deletion) syndrome (with hypocalcemia and Grave's disease), Williams-Beuren syndrome (with hypercalcemia, DM, and hypothyroidism), and Down syndrome (with thyroid disorders and DM). More commonly, ACHD is associated with abnormal glucose metabolism (AGM) including insulin resistance, impaired glucose tolerance, and DM. In a study of Japanese complex ACHD patients, AGM was 10 -fold more common than the general population, and DM was present in $10 \%$ of patients. This was hypothesized as a secondary effect of ACHD-related comorbidities. Specifically, neurohormonal activation (in the context of heart failure or diuretic use), renal insufficiency, and hepatic dysfunction all are known to predispose to AGM and are commonly seen in ACHD [79]. Prevention and management of AGM in ACHD should be included in the long-term management of ACHD.

\section{Pregnancy}

Pregnancy, labor, and delivery impose significant physiologic and psychosocial challenges on CHD patients, but most can have successful courses when managed appropriately [20]. A detailed description of the management of pregnancy in ACHD is beyond the scope of this review; interested readers are directed instead to Greutmann and Pieper [80] for an excellent discussion. Many aspects of the pregnant CHD patient are lesion specific, but nearly all cases benefit from an interdisciplinary team including ACHD, subspecialty obstetrics, and obstetric anesthesia providers. Planning ideally begins with preconception and includes genetic counseling (when appropriate), risk assessment, patient and provider education, and interdisciplinary discussion of management and potential complications. Maternal complications include arrhythmias, heart failure, obstetric complications (e.g., pregnancy induced hypertension, and pre-eclampsia), infection, and thromboembolism.

\section{Conclusions}

The population of ACHD patients has grown rapidly over several decades and continued strides in their treatment portend further growth. Increasing clinical experience and research has illuminated the many extra-cardiac manifestations and complications of ACHD and highlighted its nature as a systemic disease. ACHD patients are, therefore, best served by a multidisciplinary approach most often found at 
tertiary care centers and by specialists whose expertise extends beyond structural heart disease.

Adult-medicine providers are best able to care for the many adult-specific aspects of ACHD care. Systemic complications arising over a lifetime of CHD differ from those in a younger population. Moreover, the management of nearly any noncardiac, adult pathology is influenced by (or often the result of) concurrent CHD. Finally, increased survival into adulthood means more ACHD patients require routine adult health maintenance programs best implemented by adult providers.

The prevalence and risk factors for many extra-cardiac manifestations of ACHD have now been identified, but ACHD-specific strategies for their prevention or management remain a needed focus for future research.

\section{R E F E R E N C E S}

[1] Williams RG, Pearson GD, Barst RJ, Child JS, del Nido P, Gersony WM, et al. Report of the National Heart, Lung, and Blood Institute Working Group on research in adult congenital heart disease. J Am Coll Cardiol 2006;47:701-7.

[2] Marelli AJ, Ionescu-Ittu R, Mackie AS, Guo L, Dendukuri N, Kaouache M. Lifetime prevalence of congenital heart disease in the general population from 2000 to 2010. Circulation 2014;130:749-56.

[3] Mazor Dray E, Marelli AJ. Adult congenital heart disease. Cardiol Clin 2015;33:503-12.

[4] Verheugt CL, Uiterwaal CSPM, van der Velde ET, Meijboom FJ, Pieper PG, Sieswerda GT, et al. The emerging burden of hospital admissions of adults with congenital heart disease. Heart 2010;96:872-8.

[5] Lanz J, Brophy JM, Therrien J, Kaouache M, Guo L, Marelli AJ. Stroke in adults with congenital heart disease: incidence, cumulative risk and predictors. Circulation 2015. http://dx.doi. org/10.1161/CIRCULATIONAHA.115.011241-6.

[6] Bannan A, Shen R, Silvestry FE, Herrmann HC. Characteristics of adult patients with atrial septal defects presenting with paradoxical embolism. Cathet Cardiovasc Intervent 2009;74:1066-9.

[7] Warnes CA, Williams RG, Bashore TM, Child JS, Connolly HM, Dearani JA, et al. ACC/AHA 2008 guidelines for the management of adults with congenital heart disease: a report of the American College of Cardiology/American Heart Association Task Force on practice guidelines (writing committee to develop guidelines on the management of adults with congenital heart disease): developed in collaboration with the American Society of Echocardiography, Heart Rhythm Society, International Society for Adult Congenital Heart Disease, Society for Cardiovascular Angiography and Interventions, and Society of Thoracic Surgeons. Circulation 2008;118: e714-833.

[8] Rohrhoff N, Vavalle JP, Halim S, Kiefer TL, Harrison JK. Current status of percutaneous PFO closure. Curr Cardiol Rep 2014;16:477-9.

[9] Connolly HM, Huston J, Brown RD, Warnes CA, Ammash NM, Tajik AJ. Intracranial aneurysms in patients with coarctation of the aorta: a prospective magnetic resonance angiographic study of 100 patients. Mayo Clin Proc 2003;78: 1491-9.

[10] Donti A, Spinardi L, Brighenti M, Faccioli L, Leoni C, Fabi M, et al. Frequency of intracranial aneurysms determined by magnetic resonance angiography in children (mean age 16) having operative or endovascular treatment of coarctation of the aorta (mean age 3). Am J Cardiol 2015;116:630-3.
[11] Schievink WI, Raissi SS, Maya MM, Velebir A. Screening for intracranial aneurysms in patients with bicuspid aortic valve. Neurology 2010;74:1430-3.

[12] Goyal MS, Gottumukkala R, Bhalla S, Kates A, Zipfel GJ, Derdeyn CP. Bicuspid aortic valves and thoracic aortic aneurysms in patients with intracranial aneurysms. Neurology 2015;84:46-9.

[13] Jensen AS, Idorn L, Thomsen C, Recke von der P, Mortensen J, Sørensen KE, et al. Prevalence of cerebral and pulmonary thrombosis in patients with cyanotic congenital heart disease. Heart 2015;101:1540-6.

[14] Jensen AS, Johansson PI, Idorn L, Sørensen KE, Thilén U, Nagy E, et al. The haematocrit-an important factor causing impaired haemostasis in patients with cyanotic congenital heart disease. Int J Cardiol 2013;167:1317-21.

[15] Fischbein CA, Rosenthal A, Fischer EG, Nadas AS, Welch K. Risk factors of brain abscess in patients with congenital heart disease. Am J Cardiol 1974;34:97-102.

[16] Kagawa M, Takeshita M, Yato S, Kitamura K. Brain abscess in congenital cyanotic heart disease. J Neurosurg 1983;58: 913-7.

[17] Di Filippo S. Current patterns of infective endocarditis in congenital heart disease. Heart 2006;92:1490-5.

[18] Lumbiganon P, Chaikitpinyo A. Antibiotics for brain abscesses in people with cyanotic congenital heart disease. In: Lumbiganon P, ed, Cochrane Database of Systematic Reviews, vol. 3. Chichester, UK: John Wiley \& Sons, Ltd; 2013: CD004469

[19] Tyagi M, Austin K, Stygall J, Deanfield J, Cullen S, Newman SP. What do we know about cognitive functioning in adult congenital heart disease? Cardiol Young 2014;24:13-9.

[20] Bhatt AB, Foster E, Kuehl K, Alpert J, Brabeck S, Crumb S, et al. Congenital heart disease in the older adult: a scientific statement from the American Heart Association. Circulation 2015;131:1884-931.

[21] Martinez-Biarge M, Jowett VC, Cowan FM, Wusthoff CJ. Neurodevelopmental outcome in children with congenital heart disease. Semin Fetal Neonatal Med 2013;18:279-85.

[22] Marino BS, Lipkin PH, Newburger JW, Peacock G, Gerdes M, Gaynor JW, et al. Neurodevelopmental outcomes in children with congenital heart disease: evaluation and management: a scientific statement from the American Heart Association. Circulation 2012;126:1143-72.

[23] Newburger JW. Brain injury in congenital heart disease. Circulation 2006;113:183-5.

[24] Eslami B, Sundin Ö, Macassa G, Khankeh HR, Soares JJF. Anxiety, depressive and somatic symptoms in adults with congenital heart disease. J Psychosom Res 2013;74:49-56.

[25] Kovacs AH, Utens EM. More than just the heart. Cardiol Clin 2015;33:625-34.

[26] Kovacs AH, Moons P. Psychosocial functioning and quality of life in adults with congenital heart disease and heart failure. Heart Fail Clin 2014;10:35-42.

[27] Kourkoveli P, Rammos S, Parissis J, Maillis A, Kremastinos D, Paraskevaidis I. Depressive symptoms in patients with congenital heart disease: incidence and prognostic value of self-rating depression scales. Congenit Heart Dis 2015;10: 240-7.

[28] Müller J, Hess J, Hager A. Minor symptoms of depression in patients with congenital heart disease have a larger impact on quality of life than limited exercise capacity. Int J Cardiol 2012;154:265-9.

[29] Connolly D, McClowry S, Hayman L, Mahony L, Artman M. Posttraumatic stress disorder in children after cardiac surgery. J Pediatr 2004;144:480-4.

[30] Horner T, Liberthson R, Jellinek MS. Psychosocial profile of adults with complex congenital heart disease. Mayo Clin Proc 2000;75:31-6. 
[31] Diller GP. Exercise intolerance in adult congenital heart disease: comparative severity, correlates, and prognostic implication. Circulation 2005;112:828-35.

[32] Ginde S, Bartz PJ, Hill GD, Danduran MJ, Biller J, Sowinski J, et al. Restrictive lung disease is an independent predictor of exercise intolerance in the adult with congenital heart disease. Congenit Heart Dis 2013;8:246-54.

[33] Alonso-Gonzalez R, Borgia F, Diller GP, Inuzuka R, Kempny A, Martinez-Naharro A, et al. Abnormal lung function in adults with congenital heart disease: prevalence, relation to cardiac anatomy, and association with survival. Circulation 2013; 127:882-90.

[34] Pianosi PT, Johnson JN, Turchetta A, Johnson BD. Pulmonary function and ventilatory limitation to exercise in congenital heart disease. Congenit Heart Dis 2009;4:2-11.

[35] Healy F, Hanna BD, Zinman R. Pulmonary complications of congenital heart disease. Paediatr Respir Rev 2012;13:10-5.

[36] Lowe BS, Therrien J, Ionescu-Ittu R, Pilote L, Martucci G, Marelli AJ. Diagnosis of pulmonary hypertension in the congenital heart disease adult population. J Am Coll Cardiol 2011;58:538-46.

[37] Simonneau G, Gatzoulis MA, Adatia I, Celermajer D, Denton C, Ghofrani A, et al. Updated clinical classification of pulmonary hypertension. J Am Coll Cardiol 2013;62:D34-41.

[38] Gupta V, Tonelli AR, Krasuski RA. Congenital heart disease and pulmonary hypertension. Heart Fail Clin 2012;8:427-45.

[39] Diller GP, Gatzoulis MA. Pulmonary vascular disease in adults with congenital heart disease. Circulation 2007;115:1039-50.

[40] Opotowsky AR. Clinical evaluation and management of pulmonary hypertension in the adult with congenital heart disease. Circulation 2015;131:200-10.

[41] Krieger EV, Fernandes SM. Heart failure caused by congenital left-sided lesions. Heart Fail Clin 2014;10:155-65.

[42] Asrani SK, Asrani NS, Freese DK, Phillips SD, Warnes CA, Heimbach J, et al. Congenital heart disease and the liver. Hepatology 2012;56:1160-9.

[43] Nawrocki P, Wesnerowicz A, Schmidt C, Malec E, Januszewska K. Scimitar syndrome associated with abnormal hepatic venous drainage. World J Pediatr Congenit Heart Surg 2015; 6:474-6.

[44] Ford RM, Book W, Spivey JR. Liver disease related to the heart. Transplant Rev 2015;29:33-7.

[45] Wu FM, Opotowsky AR, Raza R, Harney S, Ukomadu C, Landzberg MJ, et al. Transient elastography may identify Fontan patients with unfavorable hemodynamics and advanced hepatic fibrosis. Congenit Heart Dis 2014;9:438-47.

[46] Jalal Z, Iriart X, De Lédinghen V, Barnetche T, Hiriart J-B, Vergniol J, et al. Liver stiffness measurements for evaluation of central venous pressure in congenital heart diseases. Heart 2015;101:1499-504.

[47] Shiina Y, Toyoda T, Kawasoe Y, Tateno S, Shirai T, Matsuo $\mathrm{K}$, et al. The prevalence and risk factors for cholelithiasis and asymptomatic gallstones in adults with congenital heart disease. Int J Cardiol 2011;152:171-6.

[48] Mattar W, Juliar B, Gradus-Pizlo I, Kwo PY. Amiodarone hepatotoxicity in the context of the metabolic syndrome and right-sided heart failure. J Gastrointestin Liver Dis 2009;18:419-23.

[49] Wang A, Book WM, McConnell M, Lyle T, Rodby K, Mahle WT. Prevalence of hepatitis C infection in adult patients who underwent congenital heart surgery prior to screening in 1992. Am J Cardiol 2007;100:1307-9.

[50] McCabe N, Farris AB, Hon H, Ford R, Book WM. Hepatocellular carcinoma in an adult with repaired tetralogy of fallot. Congenit Heart Dis 2013;8:E139-44.

[51] Izumi $Y$, Hiramatsu $N$, Itose $I$, Inoue $T$, Sasagawa $A$, Egawa S, et al. Juvenile hepatocellular carcinoma with congestive liver cirrhosis. J Gastroenterol 2005;40:204-8.
[52] Wu FM, Ukomadu C, Odze RD, Valente AM, Mayer JE, Earing MG. Liver disease in the patient with Fontan circulation. Congenit Heart Dis 2011;6:190-201.

[53] Te HS, Anderson AS, Millis JM, Jeevanandam V, Jensen DM. Current state of combined heart-liver transplantation in the United States. J Heart Lung Transplant 2008;27:753-9.

[54] Hollander SA, Reinhartz O, Maeda K, Hurwitz M, N Rosenthal D, Bernstein D. Intermediate-term outcomes after combined heart-liver transplantation in children with a univentricular heart. J Heart Lung Transplant 2013;32:368-70.

[55] Robinson JA, Driscoll DJ, O'Leary PW, Burkhart HM, Dearani JA, Daly RC, et al. Cardiac and multiorgan transplantation for end-stage congenital heart disease. Mayo Clin Proc 2014;89:478-83.

[56] Cannon RM, Hughes MG, Jones CM, Eng M, Marvin MR. A review of the United States experience with combined heart-liver transplantation. Transpl Int 2012;25:1223-8.

[57] Kiesewetter CH, Sheron N, Vettukattill JJ, Hacking N, Stedman B, Millward-Sadler $\mathrm{H}$, et al. Hepatic changes in the failing Fontan circulation. Heart 2007;93:579-84.

[58] Dimopoulos K, Diller GP, Koltsida E, Pijuan-Domenech A, Papadopoulou SA, Babu-Narayan SV, et al. Prevalence, predictors, and prognostic value of renal dysfunction in adults with congenital heart disease. Circulation 2008; 117:2320-8.

[59] Pankau R, Partsch CJ, Winter M, Gosch A, Wessel A. Incidence and spectrum of renal abnormalities in Williams-Beuren syndrome. Am J Med Genet 1996;63:301-4.

[60] Morgan C, Al-Aklabi M, Garcia Guerra G. Chronic kidney disease in congenital heart disease patients: a narrative review of evidence. Can J Kidney Health Dis 2015;2:163-9.

[61] Flanagan MF, Hourihan M, Keane JF. Incidence of renal dysfunction in adults with cyanotic congenital heart disease. Am J Cardiol 1991;68:403-6.

[62] Khairy P, Landzberg MJ. Adult congenital heart disease: toward prospective risk assessment of a multisystemic condition. Circulation 2008;117:2311-2.

[63] Hsu R-B, Tsai M-K, Lee P-H, Lee C-M, Chen M-F, Wang S-S, et al. Simultaneous heart and kidney transplantation from a single donor. Eur J Cardiothorac Surg 2008;34:1179-84.

[64] Giglia TM, Massicotte MP, Tweddell JS, Barst RJ, Bauman M, Erickson CC, et al. Prevention and treatment of thrombosis in pediatric and congenital heart disease: a scientific statement from the American Heart Association. Circulation 2013;128:2622-703.

[65] Collins N, Piran S, Harrison J, Azevedo E, Oechslin E, Silversides CK. Prevalence and determinants of anemia in adults with complex congenital heart disease and ventricular dysfunction (subaortic right ventricle and single ventricle physiology). Am J Cardiol 2008;102:625-8.

[66] Dimopoulos K, Diller G-P, Giannakoulas G, Petraco R, Chamaidi A, Karaoli E, et al. Anemia in adults with congenital heart disease relates to adverse outcome. J Am Coll Cardiol 2009;54:2093-100.

[67] Broberg CS, Jayaweera AR, Diller GP, Prasad SK, Thein SL, Bax BE, et al. Seeking optimal relation between oxygen saturation and hemoglobin concentration in adults with cyanosis from congenital heart disease. Am J Cardiol 2011;107:595-9.

[68] Kaemmerer H, Fratz S, Braun SL, Koelling K, Eicken A, Brodherr-Heberlein S, et al. Erythrocyte indexes, iron metabolism, and hyperhomocysteinemia in adults with cyanotic congenital cardiac disease. Am J Cardiol 2004;94:825-8.

[69] Broberg CS, Bax BE, Okonko DO, Rampling MW, Bayne S, Harries C, et al. Blood viscosity and its relationship to iron deficiency, symptoms, and exercise capacity in adults with cyanotic congenital heart disease. J Am Coll Cardiol 2006; 48:356-65. 
[70] Martínez-Quintana E, Rodríguez-González F. Iron deficiency anemia detection from hematology parameters in adult congenital heart disease patients. Congenit Heart Dis 2013; 8:117-23.

[71] Ammash N, Warnes CA. Cerebrovascular events in adult patients with cyanotic congenital heart disease. J Am Coll Cardiol 1996;28:768-72.

[72] Tay ELW, Peset A, Papaphylactou M, Inuzuka $R$, Alonso-Gonzalez R, Giannakoulas G, et al. Replacement therapy for iron deficiency improves exercise capacity and quality of life in patients with cyanotic congenital heart disease and/or the Eisenmenger syndrome. Int J Cardiol 2011;151:307-12.

[73] Rupa-Matysek J, Trojnarska O, Gil L, Szczepaniak-Chicheł L, Wojtasińska E, Tykarski A, et al. Assessment of coagulation profile by thromboelastometry in adult patients with cyanotic congenital heart disease. Int J Cardiol 2016;202:556-60.

[74] Waldow HC, Westhoff-Bleck M, Widera C, Templin C, Depka, von M. Acquired von Willebrand syndrome in adult patients with congenital heart disease. Int J Cardiol 2014; $176: 739-45$
[75] Lill MC, Perloff JK, Child JS. Pathogenesis of thrombocytopenia in cyanotic congenital heart disease. Am J Cardiol. 2006;98:254-8.

[76] Rosove MH, Perloff JK, Hocking WG, Child JS, Canobbio MM, Skorton DJ. Chronic hypoxaemia and decompensated erythrocytosis in cyanotic congenital heart disease. Lancet 1986;2:313-5.

[77] Reiss UM, Bensimhon P, Zimmerman SA, Ware RE. Hydroxyurea therapy for management of secondary erythrocytosis in cyanotic congenital heart disease. Am J Hematol 2007;82: 740-3.

[78] Mortensen KH, Andersen NH, Gravholt CH. Cardiovascular phenotype in Turner syndrome-integrating cardiology, genetics, and endocrinology. Endocr Rev 2012;33: 677-714.

[79] Ohuchi H, Miyamoto Y, Yamamoto M, Ishihara H, Takata H, Miyazaki A, et al. High prevalence of abnormal glucose metabolism in young adult patients with complex congenital heart disease. Am Heart J 2009;158:30-9.

[80] Greutmann M, Pieper PG. Pregnancy in women with congenital heart disease. Eur Heart J 2015;36:2491-9. 